\title{
Conocimiento y prácticas de bioseguridad en la canalización periférica vía venosa en estudiantes de enfermería
}

\author{
Miluska Murillo-Reyes ${ }^{1}$ \\ Sara Huamán-Lázaro²
}

RESUMEN: El objetivo fue determinar los niveles del conocimiento y de la práctica sobre las prácticas de bioseguridad en la canalización de vía venosa periférica en 39 estudiantes de enfermería de una universidad privada de Lima Norte. Se emplearon porcentajes y Chi - cuadrado con una significancia estadística menor o igual a 0.05 . El 54\% tuvieron un conocimiento alto sobre medidas de bioseguridad en la canalización de vía venosa periférica y un $74 \%$ realizó una práctica adecuada. Tener una práctica medianamente adecuada sobre medidas de bioseguridad en la canalización de vía venosa periférica se asoció/coincidió con tener un conocimiento alto de la misma en los estudiantes de enfermería. Se sugiere que los estudiantes sigas las pautas de la guía práctica, asistir a las capacitaciones y realizar las prácticas hospitalarias de manera continua.

PALABRAS CLAVE: Exposición a Agentes Biológicos; Alcantarillado; Conocimiento; Estudiantes de enfermería.

Citar como: Murillo M, Huamán S. Conocimiento y prácticas de bioseguridad en la canalización periférica vía venosa en estudiantes de enfermería. CASUS. 2017; 2(2):pp. 


\section{INTRODUCCIÓN}

En la actualidad se vienen dando casos de accidentes intrahospitalarios en diversos países debido a una mala praxis de estudiantes de Enfermería. Este evento, puede facilitar la adquisición de alguna enfermedad infectocontagiosa tanto en el paciente como en mismo estudiante. Por otra parte, esta situación puede originar desconfianza de algunos profesionales de la salud que laboran de manera estable frente a los estudiantes practicantes limitando las posibilidades de realizar prácticas hospitalarias (1).

Un estudio revela datos de accidentes ocurridos por malas prácticas en bioseguridad en estudiantes universitarios de Enfermería en distintos países. Por ejemplo, en España se presentó un $42.2 \%$ de estos accidentes, un $72.2 \%$ en Turquía, un $74.7 \%$ en Chile y un $47.5 \%$ en Brasil (1). Por este motivo se suele limitar la participación del estudiante debido a que los enfermeros profesionales a cargo del servicio tienen la percepción de que el estudiante podría incurrir en una inadecuada praxis por su poca experiencia profesional (2). Los accidentes laborales registrados son producidos por contacto directo, con fluidos biológicos sin haber tomado en cuenta las medidas de bioseguridad y por accidentes punzocortantes (3).

Por otra parte, la canalización de vía venosa periférica es el procedimiento más utilizado de enfermería sirviendo como vía medicamentosa más eficaz de administración por la absorción casi inmediata del medicamento. Este procedimiento consta en la inserción de un catéter en el área venosa; por ello, el buen conocimiento de esta proporcionará mayor bioseguridad, mayor tiempo de duración, fácil acceso y permeabilidad. Así mismo, permitirá disminuir los riesgos de una posible infección tanto en el paciente como el profesional de enfermería. Con lo anterior se comprende por qué la práctica clínica es el rubro más importante para poder adquirir experiencia y aplicar los conocimientos teóricos (4).

Este procedimiento es el más enseñado en la vida universitaria tanto desde la teoría como en la práctica. El estudiante cuando llega a ciclos superiores alcanza madurez emocional y desea tener un pensamiento crítico. A este nivel opta no sólo por quedarse con el conocimiento que le brinda el docente, sino que, por iniciativa propia, busca su mejoría. Con lo anterior se pone en acto lo que llamamos autoaprendizaje y como consecuencia se desarrollan las competencias del estudiante (5).
Un estudio muestra, a partir de una evaluación, la práctica da canalización de vía periférica venosa en internos de enfermería reportando asociación entre su nivel medio y un conocimiento alto (6). Asimismo, los estudiantes más jóvenes y los que se encuentran en los últimos ciclos universitarios tienen un buen conocimiento $(7,8)$.

Teniendo en cuenta la relevancia formativa de las prácticas clínicas para los estudiantes de Enfermería, la frecuente utilización de la canalización venosa y los escasos estudios nacionales de evaluación del conocimiento y práctica sobre las medidas de bioseguridad en la canalización de vía venosa periférica se planeó como objetivo: determinar los niveles de conocimiento y de la práctica sobre las medidas de bioseguridad en la canalización de vía venosa periférica en estudiantes de Enfermería.

\section{MATERIALES Y MÉTODOS}

El estudio fue transversal descriptivo y correlacional. La muestra fue de 39 estudiantes de Enfermería de una universidad privada de Lima Norte. El muestreo fue censal, incluyéndose alumnos de ambos sexos de los ciclos 5to, 6to, 7mo, 8vo e internado. Se excluyeron aquellos que no habías realizado prácticas continuas de la canalización de vía venosa periférica y que no estudiaban en la carrera de Enfermería.

Para evaluar la variable conocimiento sobre medidas de bioseguridad en la canalización de vía venosa periférica (variable categórica ordinal) fue categorizada como bajo (3-0 puntos), medio (8-4 puntos) y alto (12-9 puntos). Se utilizó una encuesta validada a nivel nacional con 12 preguntas acerca del manejo de la bioseguridad en la canalización de vía periférica: antes ( 6 preguntas), durante ( 2 preguntas) y después (4 preguntas) (6).

La variable práctica del manejo sobre medidas de bioseguridad en la canalización de vía venosa periférica (variable ordinal) fue categorizada como inadecuada (0-7 puntos), medianamente adecuada (816 puntos) y adecuada (17-28 puntos). Fue medida por una lista de chequeo validada a nivel nacional que constaba de 28 enunciados clasificados en tres dimensiones: antes del procedimiento (13 enunciados), después del procedimiento (10 enunciados) y después del procedimiento (5 enunciados) (4). Las variables sociodemográficas fueron: edad, variable categórica $(18-20 ; 21-25 ; 26-$ 30 ; >30); sexo, variable categórica (femenino y masculino); estado civil, variable categórica (soltero, 
casado y conviviente); residencia, variable categórica (urbano y rural); carrera técnica de Enfermería, variable categórica (sí y no); Trabajo actual, variable categórica (sí y no); trabajo actual en un establecimiento de salud, variable categórica (sí y no) y tipo de contrato, variable categórica (contratado y nombrado).

Se utilizó el programa STATA 12 para la realización del análisis estadístico. Se calcularon porcentajes para las variables categóricas. Respecto al análisis bivariado se utilizó la prueba estadística Chi cuadrado con una significancia estadística menor o igual a 0.05 .

Para la realización del estudio los datos obtenidos fueron aprobados por el Comité de Ética Institucional. Asimismo, se respetó la confidencialidad manifestada en el consentimiento informado. Además, no hubo algún riesgo para la salud de los participantes.

\section{RESULTADOS}

De los 39 estudiantes de enfermería el 54\% tuvo un conocimiento alto sobre medidas de bioseguridad en la canalización de vía venosa periférica y un $74 \%$ realizó una práctica adecuada en base al manejo del mismo. La mayoría de los encuestados tenía de 21 a 25 años (54\%), fueron mujeres (90\%), no posee carrera técnica de Enfermería (82\%), trabaja actualmente (64\%), no cuenta con un trabajo actual en un centro de salud $(90 \%)$ y cursaron el Internado (44\%). No se registraron alumnos con un conocimiento bajo ni con una práctica inadecuada (ver tabla 1).

\section{Tabla 1 Descripción de las variables de la muestra}

$\mathbf{n}$

Nivel de conocimiento
Medio
Alto

Alto

\section{Práctica}

$\begin{array}{lll}\text { Medianamente adecuada } & 10 & 26\end{array}$

Adecuada 29

74

\section{Edad}

$18-20$

$21-25$

$26-30$

$>30$

\section{Sexo}

Femenino

$35 \quad 90$

Masculino

\section{Carrera técnica de Enfermería}

Sí

$\begin{array}{ll}7 & 18\end{array}$

No

\section{Trabajo actual}

Sí

No

\section{Trabaja actual en un centro de salud}

Sí

No

Ciclo

Quinto

Sexto

Séptimo

Octavo

Internado

$3 \quad 8$

$17 \quad 44$


Tener una práctica medianamente adecuada se asoció significativamente con tener un conocimiento alto (80\%) sobre medidas de bioseguridad en la canalización de vía venosa periférica. Asimismo, tener entre 21 a 25 años (76\%), estar cursando el octavo ciclo (100\%) e Internado $(71 \%)$ se asociaron significativamente con tener un conocimiento alto del mismo (ver tabla 2).

\section{Tabla 2: Asociación descriptiva entre el conocimiento y la práctica sobre medidas de bioseguridad en la canalización de vía venosa periférica}

$\begin{array}{ccc}\text { Nivel de conocimiento } \\ \text { Regular } & \text { Alto } & \\ \mathrm{n}(\%) & \mathrm{n}(\%) & \text { p-value }\end{array}$

\section{Práctica \\ Adecuado \\ Variables \\ sociodemográficas \\ Edad}

Medianamente adecuado 20

55

80

45

0.05

$18-20$

$21-25$

$26-30$

$>30$

$71 \quad 29$

\section{Sexo}

Femenino

Masculino

\section{Carrera técnica de Enfermería} $\mathrm{Si}$

No

\section{Trabajo actual}

\section{$\mathrm{Si}$}

No

Trabaja actual en un centro de salud

$\mathrm{Si}$

No

Ciclo

Quinto

Sexto

Séptimo

Octavo

Internado

\section{DISCUSIÓN}

Los resultados muestran que tener una práctica medianamente adecuada sobre medidas de bioseguridad en la canalización de vía venosa periférica se asoció con tener un conocimiento alto de la misma en los estudiantes de Enfermería. Asimismo, tener entre los 21 y 25 años, cursar el octavo ciclo y el Internado se asociaron con tener un conocimiento alto sobre medidas de bioseguridad en la canalización de vía venosa periférica.

Tener una práctica medianamente adecuada se asoció significativamente con tener un conocimiento alto sobre medidas de bioseguridad en la canalización de vía venosa periférica. Este hallazgo es corroborado por otros estudios $(3,4)$. Los estudiantes de enfermería que ingresan a las prácticas hospitalarias con un conocimiento alto están supuestamente capacitados para realizar diversos procedimientos como es el caso de la venoclisis. Sin embargo, el no realizarlos de manera continua puede generar cierta dificultad para poder ejecutarlos con éxito en la praxis pre profesional. Por ende, en muchas ocasiones el estudiante es bueno en conocimiento, pero tiene una capacidad práctica medianamente adecuada por el déficit de oportunidades que se brinda en el hospital (2-4).

Otro hallazgo importante fue que tener entre 21 y 25 años se relacionó con poseer un conocimiento alto sobre medidas de bioseguridad en la canalización de vía venosa periférica. Otro autor confirma este hallazgo $(7,9)$. Durante la primera etapa de la juventud de los estudiantes universitarios (18 a 20 años) desencadenan diversos cambios que influyen en la capacidad de conocimiento y de razonamiento, permitiendo así mejorar su pensamiento de modo más complejo, lógico y metódico. Conforme van avanzado en sus estudios los conocimientos generados en etapas anteriores llegan a una 0.757 maduración, permitiendo al joven universitario alcanzar un mejor conocimiento a través del análisis y la crítica (10-14).

Cursar el octavo ciclo e internado se correlacionó con 0.222 un conocimiento alto sobre medidas de bioseguridad en la canalización de vía venosa periférica. Esta relación es sustentada por otras investigaciones (12, 15). Durante las últimas etapas de la formación académica universitaria los futuros enfermeros adquirieren un conjunto de motivaciones y estrategias cognitivas incentivados desde un inicio por sus docentes. Posteriormente el alumno, volviéndose más 
crítico, descubre que un autoaprendizaje es lo que le lleva a aumentar sus conocimientos y a la maduración de estos $(11,12,15)$.

Algunas variables sociodemográficas relacionadas con el conocimiento y práctica del manejo de la bioseguridad en la canalización de vía venosa periférica como el tipo de trabajo y ocupación no han sido consideradas. Las limitaciones del diseño del estudio fueron el sesgo de memoria y de selección. Se propone el desarrollo de estudios experimentales sobre el tema. Así mismo, tomar en cuenta otras variables sociodemográficas, como el número de hijos que posee el entrevistado, tiempo que se encuentra laborando, entre otros. Otras variables principales que se podrían agregar al estudio seria la actitud hacia las medidas de bioseguridad en la canalización de vía venosa periférica. Además, se sugiere aumentar las dimensiones de la población de encuestados, como también comparar entre universidades de Lima Norte.

\section{CONCLUSIONES}

Una práctica medianamente adecuada sobre medidas de la bioseguridad en la canalización de vía venosa periférica se asoció con un nivel alto de conocimiento. De tal manera que si los estudiantes tuviesen más oportunidades de practicar este procedimiento en la etapa de pre grado, su desempeño en el ámbito pre profesional podría ser mejor. Además, tener entre 21 y 25 años así como cursar ciclos superiores se asociaron con un conocimiento alto sobre medidas de bioseguridad de la misma.

Se sugiere incentivar a los alumnos de la carrera profesional de Enfermería a seguir una guía procedimental específica del manejo de la bioseguridad en la canalización de vía periférica asesorado por un docente asignado. Asimismo, que los docentes se capaciten sobre esta temática para actualizar sus conocimientos y así poder transmitirlos a los estudiantes. Es necesario el apoyo tanto de los establecimientos de salud como de las instituciones universitarias para brindar elementos necesarios de bioseguridad con la finalidad de poder realizar un correcto manejo de este procedimiento. Es importante que el estudiante de pregrado tenga prácticas continuas sobre medidas de bioseguridad en la canalización de vía venosa periférica supervisado por un docente especializado en diferentes establecimientos de salud.

\section{REFERENCIAS BIBLIOGRÁFICAS}

1. Garavito Z, Los accidentes biológicos y caracterización del riesgo en estudiantes de enfermería, Med UNAB 2012; 15(1):33-35.

2. Fernandez M, Barbosa M, Muñoz L. La enfermera clínica y las relaciones con los estudiantes de enfermería: un estudio fenomenológico, Brasil. [Tesis de licenciatura] São Paulo: Universidad de São Paulo; 2010

3. Vizcaya M, Perez, et al. Percepción de los estudiantes de enfermería sobre el entorno de aprendizaje clínico: el clima social. Evidentia 2004;1(2):8-11.

4. Cerda A. Medidas de bioseguridad que aplica el enfermero en la canalización de vía venosa periférica en el servicio de emergencia del INSN, 2011. [Tesis de especialización] Lima: Universidad Nacional Mayor de San Marcos; 2015.

5. Jusselit G. Relación entre prácticas docentes y el nivel de aprendizaje autodirigido en estudiantes de Medicina
[Revista Educativa Ciencias de la Salud] 2013;10(2):114-120.

6. Mayorca A. Conocimientos, actitudes y prácticas de medidas de bioseguridad, en la canalización de vía venosa periférica que realizan las internas de enfermería: UNMSM, 2009. [Tesis de licenciatura] Lima: Universidad Nacional Mayor de San Marcos; 2010.

7. Mansilla M. Etapas del desarrollo humano [Revista de investigación de Psicología] 2000; 3(2):105-116.

8. Narváez M. Autonomía para aprender y autonomía para vivir [Teoria de magíster] Lima: Universidad Peruana de Ciencias Aplicadas; 2003.

9. González J. ¿A qué edad madura nuestro cerebro?, [Solo sé que nada sé] Asturias: Blog;2011.

10. Serrano J. El desarrollo cognitivo del adolescente [Aprendizaje y desarrollo de la personalidad] 20012;1:1-9.
11. Osherson D, Markman E. Language and the ability to evaluate contradiction and tautologies. Cognition; 2; 1975.

12. Fierros G, Chávez A, et al. El papel de los tutores en la auto - dirección del aprendizaje en los estudiantes de Enfermería [Investigación y Educación en Enfermería] 2007;25(2):52-59.

13. Brofenbrenner F. The ecology of human development. Cambridge: Harvard University Press; 1979.

14. Rice, F. Desarrollo Humano. México: Prentice Hall Hispanoamericana S.A.; 1997

15. Universidad de Colima. Facultad de Enfermería. Curriculum para la formación de Licenciados en Enfermería. Colima, México: Universidad de Colima; 1999. p.914. 


\title{
Knowledge and practices of biosecurity measures in the peripheral vein channeling in students of nursing
}

\begin{abstract}
The objective was to determine the levels of knowledge and practice on biosecurity measures in the peripheral vein channeling in 39 nursing students of a private university in Lima Norte. Percentages and chi - squared were used with statistical significance less than or equal to $0.05 .54 \%$ had a high knowledge on biosecurity measures in the peripheral venous canalization and $74 \%$ performed an adequate practice. Having a fairly adequate practice on biosafety measures in the peripheral vein channeling was associated with having a high knowledge of it in nursing students. It is suggested that the students execute the practical guide, attend the trainings and perform their hospital practices on an ongoing basis.
\end{abstract}

KEY WORDS: Exposure to Biological Agents; Sewerage; Knowledge; Students, Nursing. 